\title{
Insuficiência tricúspide pós trauma associada a agnesia de músculo papilar anterior
}

\author{
Ronaldo Ducceschi FONTES*, Hebert Donizete SALERNO*, Serafin D. LANZIERI*, Suzana \\ Aparecida OLIVEIRA*, Jorge FERNANDEZ*, Ivana de Lamônica Freire ECKERT*, Alberto NAJJAR*, \\ Gilberto Paulo Pereira FRANCO*, Luiz César Nazário SCALA*
}

RBCCV 44205-509

\begin{abstract}
Fontes R D, Salerno H D, Lanzieri S D, Oliveira S A, Fernandez J, Eckert I L F, Najjar A, Franco G P P, Scala L C N - Insuficiência tricúspide pós trauma associada a agnesia de músculo papilar anterior. Rev Bras Cir Cardiovasc 2000; 15(3): 251-4.
\end{abstract}

RESUMO: Relatamos o caso de paciente do sexo masculino com 36 anos de idade com sinais e sintomas de insuficiência cardíaca direita. A história revelou trauma torácico há aproximadamente cinco anos. Submetido a operação para tratamento de insuficiência tricúspide, notou-se ausência do músculo papilar anterior da valva tricúspide, fenda na cúspide anterior e dilatação do anel tricuspídeo. Foi realizada sutura da fenda localizada na cúspide anterior e feita sua sustentação utilizando-se tira de pericárdio bovino fixada na face atrial e base do músculo papilar posterior. A operação foi completada com anuloplastia de Revuelta. $O$ paciente obteve nítida melhora dos sintomas no pós-operatório imediato, mantendo-se em classe funcional I (NYHA), após 22 meses de evolução.

DESCRITORES: Insuficiência da valva tricúspide, etiologia. Valva tricúspide, anormalidades. Traumatismos torácicos, complicações. Insuficiência da valva tricúspide, cirurgia. Valva tricúspide, cirurgia.

\section{INTRODUÇÃO}

A insuficiência valvar tricuspídea é freqüentemente verificada em associação a valvopatia mitral, sendo denominada insuficiência tricúspide secundária.

Outras etiologias como a endocardite infecciosa, anomalia de Ebstein, endomiocardiofibrose, doença carcinóide (originária das células de Kulchitsky no trato gastrointestinal) e o trauma fechado do tórax são geralmente menos freqüentes e, portanto, motivos de publicações com pequenas séries (1-3).

O tratamento geralmente é cirúrgico e freqüentemente conservador (3-5).
O objetivo desta comunicação é relatar o caso de um paciente com diagnóstico de insuficiência tricúspide, provocada por trauma, porém com anatomia valvar incomum.

\section{RELATO DO CASO}

Trata-se de paciente do sexo masculino, com 36 anos de idade, pardo, natural de Cuiabá-Mato Grosso, com queixa de palpitação, fadiga e aumento do volume abdominal.

A história revelou ser assintomático até há 5 anos, quando iniciou quadro de fadiga e edema de membros inferiores, sem relação com

\footnotetext{
Trabalho realizado no Serviço de Cirurgia Cardiovascular Ronaldo D. Fontes - Hospital Santa Cruz, Cuiabá, MT, Brasil.

Recebido para publicação em janeiro de 2000.

* Do Serviço de Cirurgia Cardiovascular Ronaldo D. Fontes - Cuiabá.

Endereço para correspondência: Ronaldo Ducceschi Fontes. Av. 31 de março, 212, sala 701. Cuiabá, MT, Brasil. CEP: 78020-870. Tel: (65) 622-2036. e.mail:ducceschi@terra.com.br
} 
Fontes R D, Salerno H D, Lanzieri S D, Oliveira S A, Fernandez J, Eckert I L F, Najjar A, Franco G P P, Scala L C N - Insuficiência tricúspide pós trauma associada a agnesia de músculo papilar anterior. Rev Bras Cir Cardiovasc 2000; 15(3): 251-4.

qualquer fato pregresso. Os sintomas acentuaram-se há 8 meses e o exame físico revelou grande edema de membros inferiores, taquicardia, palpitação e maior aumento do volume abdominal.

Procurou cardiologista que o medicou com diuréticos e digoxina. Obteve pouca melhora e após nova avaliação clínica, foi submetido a exames complementares, sendo em seguida encaminhado a nosso Serviço.

Ao exame físico encontrava-se em bom estado geral, estase jugular importante, com evidente pulso venoso sistólico jugular.

A ausculta pulmonar era normal. A ausculta cardíaca evidenciava sopro holossistólico no mesocárdio com manobra de Rivero-Carvalo positiva e coração arrítmico.

O fígado era palpável a $4 \mathrm{~cm}$ do rebordo costal direito e havia pulso hepático palpável. Não havia edema de membros inferiores na ocasião da internação.

A análise da radiografia do tórax demonstrava aumento global da área cardíaca.

Ao eletrocardiograma, notava-se a presença de extrassístoles ventriculares freqüentes. A ecocardiografia bidimensional revelava aumento severo de câmaras direitas, regurgitação importante através da valva tricúspide, ventrículo direito com $67 \mathrm{~mm}$ de diâmetro diastólico. Submetido a cateterismo cardíaco, cuja avaliação demonstrava ventrículo direito com hipocinesia difusa de grau leve, valva tricúspide com regurgitação importante para átrio direito, hipertensão pulmonar leve (Tabela 1), ventrículo esquerdo com função normal e artérias coronárias normais.
Com diagnóstico de insuficiência tricúspide primária, o paciente foi submetido a tratamento cirúrgico através de esternotomia mediana, instalação de circuito extracorpóreo e normotermia (Figura 1).

Aberto o átrio direito, foi identificada lesão "em fenda" na cúspide ântero-superior da valva tricúspide. Não havia músculo papilar anterior e sequer sinais de cordas tendíneas. A cúspide anterior era plana e a fenda provocava grande insuficiência (Figura 2). Realizada plástica da valva tricúspide, que consistiu de fechamento da fenda com fios de prolene 5-0 ancorados em duas tiras de pericárdio bovino, sustentação da cúspide anterior utilizando fita de pericárdio suturada na face atrial da cúspide anterior e fixada na base do músculo papilar posterior (Figura 3).

O anel tricuspídeo foi reduzido através de anuloplastia à Revuelta (2) (Figura 4).

O paciente teve boa evolução no pós-operatório imediato. Houve regressão imediata da estase jugular e do pulso hepático. A ventriculografia direita pós-operatória demonstrou insuficiência tricúspide moderada.

O paciente recebeu alta no sétimo dia de pósoperatório em classe funcional I (NYHA).

Após 22 meses de pós-operatório, foi submetido a novo estudo radiológico e ecocardiográfico, que demonstraram diminuição da área cardíaca e diâmetro diastólico de ventrículo direito diminuindo para $57 \mathrm{~mm}$ (10 $\mathrm{mm}$ menos que no pré-operatório) e com insuficiência tricúspide leve a moderada. $\mathrm{O}$ paciente encontra-se assintomático, com pulso hepático ausente, sem estase jugular, apresenta ritmo sinusal, entretanto ainda medicado com amiodarona.

TABELA 1

VALORES PRESSÓRICOS DAS CAVIDADES CARDÍACAS OBTIDOS NO CATETERISMO PRÉ E PÓS-OPERATÓRIO

\begin{tabular}{ccccccc}
\hline PRESSÃO $\mathbf{~ m m ~ H g / ~}$ & \multicolumn{2}{c}{ PRÉ } & & \multicolumn{2}{c}{ PÓs } \\
\hline CAVIDADE & S & D & M & S & D & M \\
AD & - & - & 25 & - & - & 8 \\
VD & 45 & 18 & 28 & 33 & 14 & 20,0 \\
TP & 45 & 18 & 27 & 33 & 14 & 20,3 \\
VE & 140 & 0 & 20 & - & - & - \\
CP & - & - & 20 & - & - & - \\
Onda "v" AD & - & 35 & - & - & - \\
\hline
\end{tabular}

AD: átrio direito; VD: ventrículo direito; TP: tronco pulmonar; VE: ventrículo esquerdo; CP: capilar pulmonar; S: sistólica; D: diastólica; M: média. 
Fontes R D, Salerno H D, Lanzieri S D, Oliveira S A, Fernandez J, Eckert I L F, Najjar A, Franco G P P, Scala L C N - Insuficiência tricúspide pós trauma associada a agnesia de músculo papilar anterior. Rev Bras Cir Cardiovasc 2000; 15(3): 251-4.

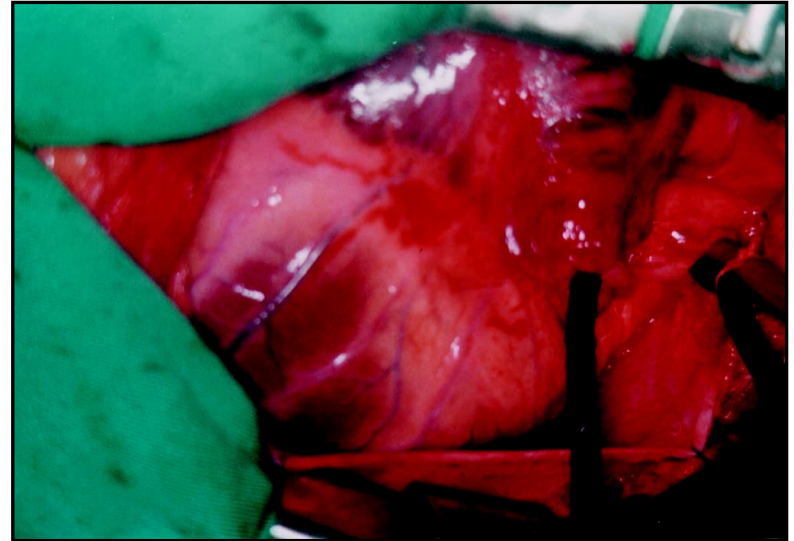

Fig. 1 - Aspecto cirúrgico que demonstra aumento das câmaras cardíacas direitas.

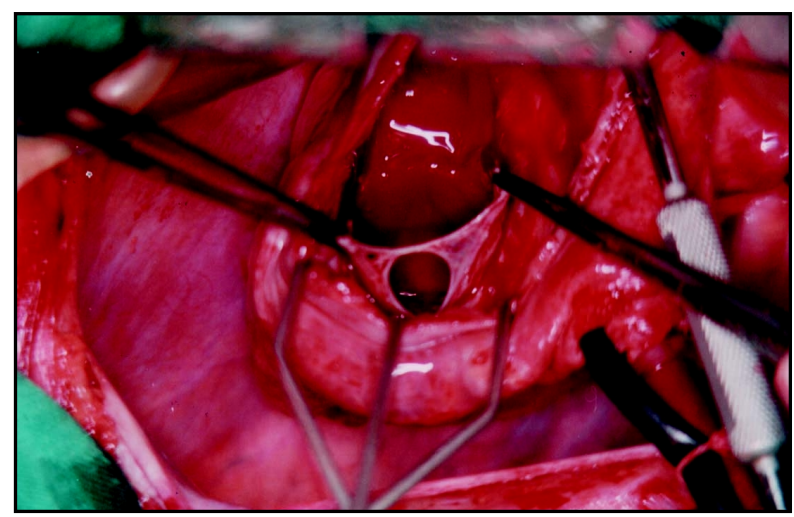

Fig. 2 - Verifica-se a cúspide anterior da valva tricúspide com a fenda central e tendíneas ausência de músculo papilar anterior e de suas cordas tendíneas.

\section{COMENTÁRIOS}

Durante a primeira avaliação clínica, não se conseguiu informações do paciente que permitissem indicar ou ao menos suspeitar sobre o real processo etiológico da insuficiência tricúspide.

Durante o ato cirúrgico, verificou-se a ausência de músculo papilar anterior da valva tricúspide, assim como das cordas tendíneas, nem mesmo vestígios da base de implantação do papilar na parede ventricular. Havia grande fenestração na cúspide anterior (Figura 2).

Motivados pelo achado cirúrgico, realizamos nova investigação sobre a história da doença, quando o paciente informou ter sofrido trauma torácico fechado, cinco anos antes da data de internação em nosso Serviço.

Revelou, ainda, que a partir do trauma, iniciaram-se os sintomas que foram se acentuando progressivamente.

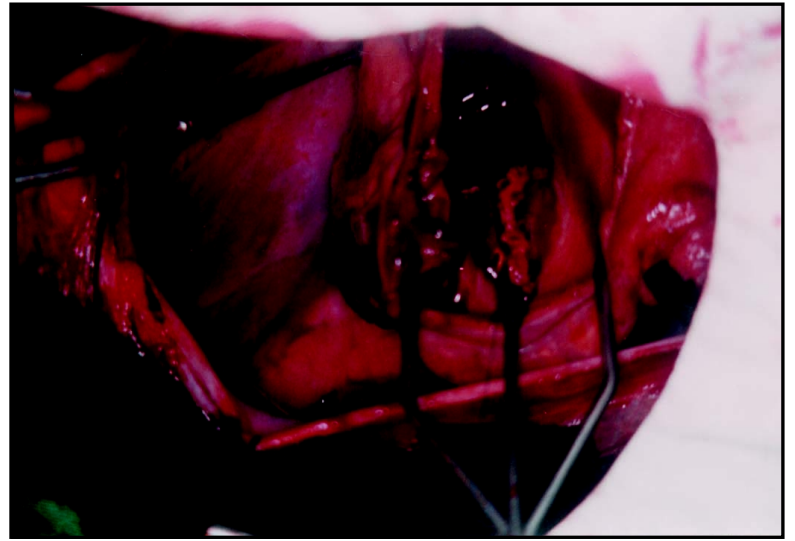

Fig. 3 - Realizada sutura da fenda utilizando-se duas tiras de pericárdio bovino.

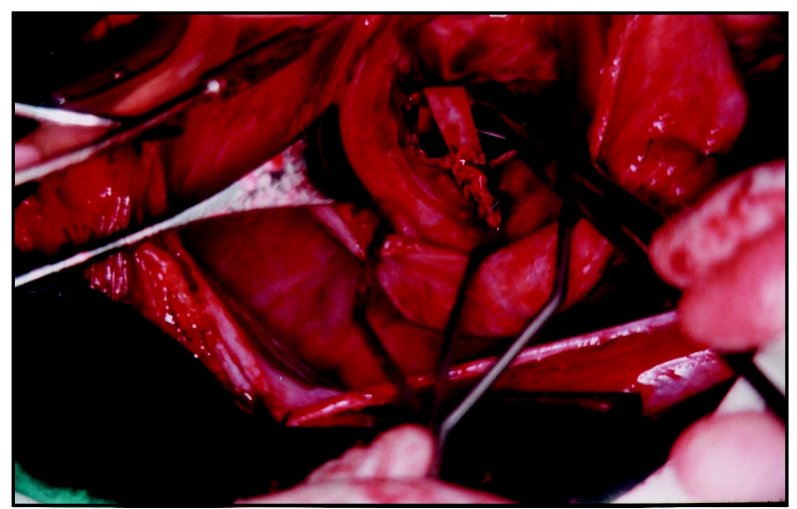

Fig. 4 - Nota-se a sutura da fenda na cúspide anterior e a plástica à Revuelta. Em detalhe a "tira" de pericárdio bovino sustentando a cúspide anterior.

Portanto, de acordo com os achados cirúrgicos, acreditamos que a ausência de músculo papilar anterior e sua cordoalha tendínea que tornavam a cúspide "plana", tenham contribuído para ocasionar insuficiência tricúspide no momento do impacto, uma vez que a valva não tinha sinais de processos degenerativos que pudessem explicar a fenestração da cúspide anterior.

As causas de insuficiência tricúspide são bem conhecidas, apesar de muitas vezes serem divulgadas como simples relato de caso (1-5).

Entretanto, a ausência do músculo papilar anterior da valva tricúspide é extremamente rara ${ }^{(6)}$. Conforme ZGHAIB et al. (7), pode, inclusive, a valva tricúspide consistir em pequeno anel sinuoso; não encontramos na literatura mais informações sobre agenesia de músculo papilar anterior da valva tricúspide.

Várias técnicas cirúrgicas podem ser realizadas 
Fontes R D, Salerno H D, Lanzieri S D, Oliveira S A, Fernandez J, Eckert I L F, Najjar A, Franco G P P, Scala L C N - Insuficiência tricúspide pós trauma associada a agnesia de músculo papilar anterior. Rev Bras Cir Cardiovasc 2000; 15(3): 251-4.

isoladamente ou em associação para o tratamento de insuficiência tricúspide, incluindo a troca valvar $(1,2,8)$.

No presente caso, associou-se algumas dessas técnicas para conservação da valva tricúspide, obtendo-se bom resultado.
O grau de insuficiência da valva tricúspide regrediu significativamente no pós-operatório imediato. Houve nítida diminuição do pulso venoso jugular e ausência do pulso hepático. Após dez meses de evolução, o paciente encontra-se assintomático.

RBCCV 44205-509

Fontes R D, Salerno H D, Lanzieri S D, Oliveira S A, Fernandez J, Eckert I L F, Najjar A, Franco G P P, Scala

$\mathrm{LCN}$ - Post-traumatic tricuspid insufficiency associated with agenesis of the anterior papillary muscle.

Rev Bras Cir Cardiovasc 2000; 15(3): 251-4

ABSTRACT: Tricuspid regurgitation arising from chest trauma five years earlier was successfully corrected by valve reconstruction in a 36 year-old man. During correction a fissure of the anterior leaflet, absence of the anterior papillary muscle, chordae tendineae and tricuspid annulus dilatation were found. Tricuspid valvuloplasty was feasible using an anchored suture of the anterior leaflet fissure, artificial bovine chordae, pericardium implantation and Revuelta ring annuloplasty. There were no complications and no early or late mortality. At 22 months follow-up tricuspid valve function has remained with mild regurgitation. The patient is in good clinical condition.

DESCRIPTORS: Tricuspid valve insufficiency, etiology. Tricuspid valve, abnormalities. Thoracic injuries, complications. Tricuspid valve insufficiency, surgery. Tricuspid valve, surgery.

\section{REFERÊNCIAS BIBLIOGRÁFICAS}

1 Leon C H, Posser M W, Rodrigues L H et al. - Insuficiência tricúspide isolada de etiologia traumática possível: relato de caso. Arq Bras Cardiol 1987; 48: 237-40.

2 Revuelta J M, Rinaldi R G, Duran C M G - Intraoperative assessment of tricuspid valve function after conservative Texas repair. Texas Heart Inst $J$ 1982; 9: 303-5.

3 Rollefson W A, Winslow T M, Adams C W, Dang M H Traumatic dehiscence of a tricuspid annuloplasty ring: diagnosis by transesophageal echocardiography. $\mathrm{Am}$ Heart J 1994; 127: 708-10.

4 Kay H J - Surgical treatment of tricuspid regurgitation. Ann Thorac Surg 1992; 53: 1132-3.

5 Chares M, Lamm P, Leischik R, Lenz G, Steinmann E H, Polonius M J - Highly acute course of ruptured papillary muscle of the tricuspid valve in a case of blunt chest trauma. J Thorac Cardiovasc Surg 1993; 41: 325-7.

6 McElhinney D B, Silverman N H, Brook M M, Hanley F L, Stanger $\mathrm{P}$ - Asymmetrically short tendinous cords causing congenital tricuspid regurgitation: improved understanding of tricuspid valvar displasia in the era of color flow echocardiography. Cardiol Young 1999; 9: 300-4.

7 Zghaib A, Attié F, Garcia Cornejo M et al. - Malformaciones congênitas de la tricúspide y anomalia de Ebstein con estenosis pulmonar valvar. Arch Inst Cardiol Mex 1981; 51: 331-9.

8 Stolf N A G, Moreira L F P, Costa R et al. - Tratamento cirúrgico conservador na insuficiência tricúspide funcional. Rev Hosp Clín Fac Med S Paulo 1981; 36: 146-51. 